\title{
Spin-orbit Hamiltonian for organic crystals from first-principles electronic structure and Wannier functions
}

\author{
Subhayan Roychoudhury and Stefano Sanvito \\ School of Physics, AMBER and CRANN Institute, Trinity College Dublin, Dublin 2, Ireland
}

(Received 25 November 2016; published 21 February 2017)

\begin{abstract}
Spin-orbit coupling in organic crystals is responsible for many spin-relaxation phenomena, going from spin diffusion to intersystem crossing. With the goal of constructing effective spin-orbit Hamiltonians to be used in multiscale approaches to the thermodynamical properties of organic crystals, we present a method that combines density functional theory with the construction of Wannier functions. In particular we show that the spin-orbit Hamiltonian constructed over maximally localized Wannier functions can be computed by direct evaluation of the spin-orbit matrix elements over the Wannier functions constructed in absence of spin-orbit interaction. This eliminates the problem of computing the Wannier functions for almost degenerate bands, a problem always present with the spin-orbit-split bands of organic crystals. Examples of the method are presented for isolated organic molecules, for monodimensional chains of $\mathrm{Pb}$ and $\mathrm{C}$ atoms and for triarylamine-based one-dimensional single crystals.
\end{abstract}

DOI: 10.1103/PhysRevB.95.085126

\section{INTRODUCTION}

Spintronics devices operate by detecting the spin of a carrier in the same way as a regular electronic device measures its electrical charge [1]. These devices are already the state of the art in the design of magnetic sensors such as the magnetic readhead of hard-disk drives [2], but also have excellent prospect as logic gate elements [3-6]. Logic circuits using the spin degree of freedom may offer low energy consumption and high speed owing to the fact that the dynamics of spins takes place at a much smaller energy scale than that of the charge $[1,3]$.

Recent years have also witnessed a marked increase in interest into investigations of organic molecules and molecular crystals as materials platform, initially for electronics $[7,8]$ and lately also for spintronics [9-11]. The main reason behind such interest is that organic crystals, coming in a wide chemical variety, are typically much more flexible than their inorganic counterparts and they can exhibit an ample range of electronic properties, which are highly tuneable in practice. For example, it is possible to change the conductivity of organic polymers over 15 orders of magnitude [12]. In addition to such extreme spectrum of physical/chemical properties organic materials are usually processed at low temperature. This is an advantage over inorganic compounds, which translates into a drastic reduction of the typical manufacturing and infrastructure costs [13]. Finally, specific to spintronics is the fact that both the spin-orbit (SO) and hyperfine interaction are very weak [14] in organic compounds, resulting in a weak spin scattering during the electron transport [15-17].

Regardless of the type of media used, either organic or inorganic, spintronics always concerns phenomena related to the injection, manipulation, and detection of spins into a solid state environment [11]. In the prototypical spintronic device, the spin valve [18], a nonmagnetic spacer, is sandwiched between two ferromagnets. Spins, which are initially aligned along the magnetization vector of the first ferromagnet, travel to the other ferromagnet through the spacer, and the resistance of the entire device depends on the relative orientation of the magnetization vectors of the two magnets. However, if the spin direction is lost across the spacer, the resistance will become independent of the magnetic configuration of the device. As such, in order to measure any spin-dependent effect one has to ensure that the charge carriers maintain their spin direction through the spacer. Notably, this requirement is not only demanded by spin valves, but also by any devices based on spins. There are several mechanisms for spin relaxation in the solid state [19].

In an organic semiconductor (OSC) the unwanted spin relaxation can be caused by the presence of paramagnetic impurities, by SO coupling, and by hyperfine interaction. In general paramagnetic impurities can be controlled to a very high degree of precision and they can be almost completely eliminated from an OSC during the chemical synthesis [20]. The hyperfine interaction instead can be usually considered small. This is because there are only a few elements typically present in organic molecules with abundant isotopes baring nuclear spins. The most obvious exception is hydrogen. However, most of the OSC crystals are $\pi$ conjugated and the $\pi$ states, responsible for the extremal energy levels, and hence for the electron transport, are usually delocalized. This means that the overlap of the wave function over the $\mathrm{H}$ nuclei has to be considered small. Finally, also the SO coupling is weak owing to the fact that most of the atoms composing organic compounds are light.

As such, since all the non-spin-conserving interactions are weak in OSCs, it is not surprising that there is contradictory evidence concerning the interaction mostly responsible for spin diffusion in organic crystals. Conflicting experimental evidence exists supporting either the SO coupling [21,22] or the hyperfine interaction $[23,24]$, indicating that the dominant mechanism may depend on the specific material under investigation. For this reason it is important to develop methods for determining the strength of both the SO and the hyperfine coupling in real materials. These can eventually be the basis for constructing effective Hamiltonians to be used for the evaluation of the relevant thermodynamics quantities (e.g., the spin diffusion length). Here we present one of such methods for the case of the SO interaction.

The SO interaction is a relativistic effect arising from the electron motion in the nuclear potential. In the electron 
reference frame the nucleus moves and creates a magnetic field, which in turn interacts with the electron spin. This is the spin-orbit coupling [25]. Since the SO interaction allows the spin of an electron to change direction during the electron motion, it is an interaction responsible for spin relaxation. In fact, there exist several SO-based microscopic theories of spin relaxation in solid state systems [19]. In the case of inorganic semiconductors these usually require knowledge of the band structure of the material, some information about its mobility, and an estimate of the spin-orbit strength. In the case of OSCs the situation, however, is more complex, mostly because the transport mechanism is more difficult to describe. First, the band picture holds true only for a few cases, while for many others one has to consider the material as an ensemble of weakly coupled molecules with a broad distribution of hopping integrals [26]. Second, the typical phonon energies are of the same order of magnitude of the electronic bandwidth, indicating that electron-phonon scattering cannot be treated as a perturbation of the band structure. For all these reasons the description of the thermodynamical properties of OSCs requires the construction of a multiscale theory, where the elementary electronic structure is mapped onto an effective Hamiltonian retaining only a handful of the original degrees of freedom [27]. A rigorous and now standard method for constructing such an effective Hamiltonian consists in calculating the band structure over a set of Wannier functions [28,29]. These can be constructed in a very general way as the Fourier transform of a linear combination of Bloch states, where the linear combination is taken so to minimize the spatial extension of the Wannier functions. These are the so-called maximally localized Wannier functions (MLWFs) [30,31].

The MLWF method performs best for well-isolated bands. This is indeed the case of OSCs, where often the valence and conduction bands originate respectively from the highest occupied molecular orbital (HOMO) and the lowest unoccupied molecular orbital (LUMO) of the gas-phase molecule. In fact, when the MLWF procedure is applied to such band structure one obtains Wannier orbitals almost identical to the molecule HOMO and LUMO [27]. Spin-orbit interaction, however, splits such well-defined bands, and in OSCs the split is typically a few tenths of $\mu \mathrm{eV}$. Thus, in this case, one has to apply the MLWF procedure to bands, which are indistinguishable at an energy scale larger then a few $\mu \mathrm{eV}$. In such conditions the minimization becomes almost impossible to converge, the MLWFs cannot be calculated for SO-split bands and an alternative scheme must be implemented.

Here we describe a method for obtaining the SO matrix elements with respect to the Wannier functions calculated in the absence of the SO interaction. Since the SO coupling in OSCs is weak, such spin-independent Wannier functions represent a close approximation of those that one could, at least in principle, obtain in the presence of the SO interaction. Furthermore, when the MLWF basis spans the same Hilbert space defined by all the atomic orbitals relevant for describing a given bands manifold, our method provides an accurate description of the system even in the case of heavy elements, i.e., for strong spin-orbit interaction. In particular we implement our scheme together with the atomic-orbital, pseudopotential, density functional theory (DFT) code SIESTA [32]. SIESTA is used to generate the band structure in absence of the spin-orbit interaction and for calculating the SO potential, while the MLWF procedure is performed with the WANNIER90 code [33].

The paper is organized as follows. In the next section we describe our method in detail, by starting from the general idea and then going into the specific numerical implementation. A how-to workflow will also be presented. Next we discuss results obtained for rather diverse physical systems. First, we evaluate the SO-split energy eigenvalues of a plumbane molecule and show how accurately these match those obtained directly from DFT including SO interaction. Then, we apply our procedure to the calculation of the band structure of a chain of $\mathrm{Pb}$ atoms, before moving to materials composed of light elements with low SO coupling. Here we will show that our method performs well for chains made of carbon atoms and of methane molecules. Finally, we obtain the SO matrix elements for the Wannier functions derived from the HOMO band of a triarylamine-based nanowire, a relatively wellknown semiconducting material with potential applications in photovoltaic [34] and spintronics.

\section{METHOD}

\section{A. General idea}

Here we describe the idea behind our method, which is general and does not depend on the specific implementation used for calculating the band structure. Consider a set of $N^{\prime}$ isolated Bloch states $\left|\psi_{m \mathbf{k}}\right\rangle$, describing an infinite lattice. These for instance can be the DFT Kohn-Sham eigenstates of a crystal. One can then obtain the associated $N^{\prime}$ Wannier functions from the definition

$$
\left|w_{n \mathbf{R}}\right\rangle=\frac{V}{(2 \pi)^{3}} \int_{\mathrm{BZ}}\left[\sum_{m=1}^{N^{\prime}} U_{m n}^{\mathbf{k}}\left|\psi_{m \mathbf{k}}\right\rangle\right] e^{-i \mathbf{k} \cdot \mathbf{R}} d \mathbf{k},
$$

where $\left|w_{n \mathbf{R}}\right\rangle$ is the $n$th Wannier vector centered at the lattice site $\mathbf{R}, V$ is the volume of the primitive cell, and the integration is performed over the first Brillouin zone (BZ). In Eq. (1) $U^{\mathbf{k}}$ is a unitary operator that mixes the Bloch states and hence defines the specific set of Wannier functions. A particularly convenient gauge choice for $U^{\mathbf{k}}$ consists in minimizing the Wannier functions spread, which writes

$$
\Omega=\sum_{n}\left[\left\langle w_{n \mathbf{0}}\left|r^{2}\right| w_{n \mathbf{0}}\right\rangle-\left|\left\langle w_{n \mathbf{0}}|\mathbf{r}| w_{n \mathbf{0}}\right\rangle\right|^{2}\right] .
$$

Such choice defines the so-called maximally localized Wannier functions (MLWFs).

In the absence of SO coupling a Wannier function of spin $s_{1}$ is composed exclusively of Bloch states with the same spin $s_{1}$. By moving from a continuous to a discrete $k$-point representation the spin-polarized version of Eq. (1) becomes [31]

$$
\left|w_{n \mathbf{R}}^{s_{1}}\right\rangle=\frac{1}{N} \sum_{\mathbf{k}} \sum_{m} U_{m n}^{s_{1}}(\mathbf{k})\left|\psi_{m \mathbf{k}}^{s_{1}}\right\rangle e^{-i \mathbf{k} \cdot \mathbf{R}} .
$$

Note that this represents either a finite periodic lattice comprising $N$ unit cells or a sampling of $N$ uniformly distributed $k$ points in the Brillouin zone of an infinite lattice. Here the Bloch states, which are normalized within each unit cell 
according to the relation $\left\langle\psi_{m \mathbf{k}}^{s_{1}} \mid \psi_{n \mathbf{k}^{\prime}}^{s_{2}}\right\rangle=N \delta_{m, n} \delta_{\mathbf{k}, \mathbf{k}^{\prime}} \delta_{s_{1}, s_{2}}$, obey to the condition $\psi_{p \mathbf{k}}\left(\mathbf{r}_{1}\right)=\psi_{p \mathbf{k}}\left(\mathbf{r}_{N+1}\right)$, where $\psi_{p \mathbf{k}}\left(\mathbf{r}_{m}\right)$ denotes the Bloch function for the $p$ th band at the wave vector $\mathbf{k}$ and position $\mathbf{r}_{m}$.

The projection of a generic Bloch state onto a MLWF in the absence of SO coupling can be written as

$$
\begin{aligned}
\left\langle\psi_{q \mathbf{k}^{\prime}}^{s_{1}} \mid w_{n \mathbf{R}_{2}}^{s_{2}}\right\rangle & =\frac{1}{N} \sum_{\mathbf{k}} \sum_{m} U_{m n}^{s_{2}}(\mathbf{k})\left\langle\psi_{q \mathbf{k}^{\prime}}^{s_{1}} \mid \psi_{m \mathbf{k}}^{s_{2}}\right\rangle e^{-i \mathbf{k} \cdot \mathbf{R}} \\
& =\frac{1}{N} \sum_{\mathbf{k}} \sum_{m} U_{m n}^{s_{2}}(\mathbf{k}) e^{-i \mathbf{k} \cdot \mathbf{R}} N \delta_{q, m} \delta_{\mathbf{k}, \mathbf{k}^{\prime}} \delta_{s_{1}, s_{2}} \\
& =U_{q n}^{s_{2}}\left(\mathbf{k}^{\prime}\right) e^{-i \mathbf{k}^{\prime} \cdot \mathbf{R}} \delta_{s_{1}, s_{2}} .
\end{aligned}
$$

Hence a generic SO matrix element can be expanded over the MLWF basis set as

$$
\begin{aligned}
\left\langle w_{m \mathbf{R}_{1}}^{s_{1}}\left|\mathbf{V}_{\mathrm{SO}}\right| w_{n \mathbf{R}_{2}}^{s_{2}}\right\rangle= & \frac{1}{N^{2}} \sum_{p, q} \sum_{\mathbf{k}_{1}, \mathbf{k}_{2}}\left\langle w_{m \mathbf{R}_{1}}^{s_{1}} \mid \psi_{p \mathbf{k}_{1}}^{s_{1}}\right\rangle\left(\mathbf{V}_{\mathrm{SO}}\right)_{p \mathbf{k}_{1}, q \mathbf{k}_{2}}^{s_{1}, s_{2}} \\
& \times\left\langle\psi_{q \mathbf{k}_{2}}^{s_{2}} \mid w_{n \mathbf{R}_{2}}^{s_{2}}\right\rangle \\
= & \frac{1}{N^{2}} \sum_{p, q} \sum_{\mathbf{k}_{1}, \mathbf{k}_{2}} U_{p m}^{*\left(s_{1}\right)}\left(\mathbf{k}_{1}\right) e^{i \mathbf{k}_{1} \cdot \mathbf{R}_{1}} \\
& \times\left(\mathbf{V}_{\mathrm{SO}}\right)_{p \mathbf{k}_{1}, q \mathbf{k}_{2}}^{s_{1}, s_{2}} U_{q n}^{s_{2}}\left(\mathbf{k}_{2}\right) e^{-i \mathbf{k}_{2} \cdot \mathbf{R}_{2}}
\end{aligned}
$$

where

$$
\left(\mathbf{V}_{\mathrm{SO}}\right)_{p \mathbf{k}_{1}, q \mathbf{k}_{2}}^{s_{1}, s_{2}}=\left\langle\psi_{p \mathbf{k}_{1}}^{s_{1}}\left|\mathbf{V}_{\mathrm{SO}}\right| \psi_{q \mathbf{k}_{2}}^{s_{2}}\right\rangle .
$$

It must be noted that in the absence of SO coupling, the Bloch states are spin degenerate, i.e., there are two states corresponding to each spatial wave function, one with spin up $\left|\psi^{\uparrow}(\mathbf{r})\right\rangle=|\psi(\mathbf{r})\rangle \otimes|\uparrow\rangle$, and one with spin down $\left|\psi^{\downarrow}(\mathbf{r})\right\rangle=|\psi(\mathbf{r})\rangle \otimes|\downarrow\rangle$. The same is true for the Wannier functions, i.e., one has always the pair $\left|w^{\uparrow}(\mathbf{r})\right\rangle=|w(\mathbf{r})\rangle \otimes|\uparrow\rangle$, $\left|w^{\downarrow}(\mathbf{r})\right\rangle=|w(\mathbf{r})\rangle \otimes|\downarrow\rangle$. In the presence of SO coupling, spin mixing occurs and each Bloch and Wannier state is, in general, a linear combination of both spin vectors. Since the Bloch states (or the Wannier ones) obtained in the absence of SO coupling form a complete basis set in the Hilbert space, the SO coupling operator can be written over such basis provided that one takes both spins into account. Therefore we use such spin-degenerate states as our basis for all calculations.

\section{B. Numerical implementation}

The derivation leading to Eq. (5) is general and the final result is simply a matrix transformation of the SO operator from the basis of the Bloch states to that of Wannier ones. Note that both basis sets are those calculated in the absence of SO coupling, i.e., we have assumed that the spatial part of the basis function is not modified by the introduction of the SO interaction. For practical purposes we now wish to rewrite Eq. (5) in terms of a localized atomic-orbital basis set, i.e., we wish to make our method applicable to first-principles DFT calculations implemented over local orbitals. In particular all the calculations that will follow use the SIESTA package, which expands the wave function and all the operators over a numerical atomic-orbital basis set $\left\{\left|\phi_{\mu, \mathbf{R}_{j}}^{s}\right\rangle\right\}$, where $\left|\phi_{\mu, \mathbf{R}_{j}}^{s}\right\rangle$ denotes the $\mu$ th atomic orbital ( $\mu$ is a collective label for the principal and angular momentum quantum numbers) with spin $s$ belonging to the cell at the position $\mathbf{R}_{j}$. SIESTA uses relativistic pseudopotentials to generate the spin-orbit matrix elements with respect to the basis vectors and truncates the range of the SO interaction to the on-site terms [35]. For a finite periodic lattice comprising $N$ unit cells, a Bloch state is written with respect to atomic orbitals as

$$
\left|\psi_{p \mathbf{k}}\right\rangle=\sum_{j=1}^{N} e^{i \mathbf{k} \cdot \mathbf{R}_{j}}\left(\sum_{\mu} C_{\mu p}(\mathbf{k})\left|\phi_{\mu, \mathbf{R}_{j}}\right\rangle\right),
$$

where the coefficients $C_{\mu p}(\mathbf{k})$ are in general $\mathrm{C}$ numbers. This state is normalized over a unit cell with the allowed $\mathbf{k}$ values being $\frac{m}{N} \mathbf{K}$, where $\mathbf{K}$ is the reciprocal lattice vector and $m$ is an integer.

Hence the SO matrix elements written with respect to the spin-degenerate Bloch states calculated in absence of SO interaction are

$$
\begin{aligned}
\left\langle\psi_{p \mathbf{k}_{1}}^{s_{1}}\left|\mathbf{V}_{\mathrm{SO}}\right| \psi_{q \mathbf{k}_{2}}^{s_{2}}\right\rangle= & \sum_{j, l} e^{i\left(\mathbf{k}_{2} \cdot \mathbf{R}_{l}-\mathbf{k}_{1} \cdot \mathbf{R}_{j}\right)} \sum_{\mu, \nu} C_{\mu p}^{* s_{1}}\left(\mathbf{k}_{1}\right) C_{\nu q}^{s_{2}}\left(\mathbf{k}_{2}\right) \\
& \times\left\langle\phi_{\mu, \mathbf{R}_{j}}^{s_{1}}\left|\mathbf{V}_{\mathrm{SO}}\right| \phi_{\nu, \mathbf{R}_{l}}^{s_{2}}\right\rangle
\end{aligned}
$$

As mentioned above SIESTA neglects all the SO matrix elements between atomic orbitals located at different atoms. This leads to the approximation

$$
\left\langle\phi_{\mu, \mathbf{R}_{j}}^{s_{1}}\left|\mathbf{V}_{\mathrm{SO}}\right| \phi_{\nu, \mathbf{R}_{l}}^{s_{2}}\right\rangle=\left\langle\phi_{\mu}^{s_{1}}\left|\mathbf{V}_{\mathrm{SO}}\right| \phi_{\nu}^{s_{2}}\right\rangle \delta_{\mathbf{R}_{j}, \mathbf{R}_{l}},
$$

so that Eq. (8) becomes

$$
\begin{aligned}
& \left\langle\psi_{p \mathbf{k}_{1}}^{s_{1}}\left|\mathbf{V}_{\mathrm{SO}}\right| \psi_{q \mathbf{k}_{2}}^{s_{2}}\right\rangle \\
& \quad=\sum_{j} e^{i\left(\mathbf{k}_{2}-\mathbf{k}_{1}\right) \cdot \mathbf{R}_{j}} \sum_{\mu, v} C_{\mu p}^{*\left(s_{1}\right)}\left(\mathbf{k}_{1}\right) C_{\nu q}^{\left(s_{2}\right)}\left(\mathbf{k}_{2}\right)\left\langle\phi_{\mu}^{s_{1}}\left|\mathbf{V}_{\mathrm{SO}}\right| \phi_{\nu}^{s_{2}}\right\rangle .
\end{aligned}
$$

This can be further simplified by taking into account the relation

$$
\sum_{j=1}^{N} e^{i\left(\mathbf{k}_{1}-\mathbf{k}_{2}\right) \cdot \mathbf{R}_{j}}=N \delta_{\mathbf{k}_{1}, \mathbf{k}_{2}},
$$

which leads to the final expression for the SO matrix elements

$$
\begin{aligned}
\left\langle\psi_{p \mathbf{k}_{1}}^{s_{1}}\left|\mathbf{V}_{\mathrm{SO}}\right| \psi_{q \mathbf{k}_{2}}^{s_{2}}\right\rangle= & N \sum_{\mu, v} C_{\mu p}^{*\left(s_{1}\right)}\left(\mathbf{k}_{1}\right) C_{\nu q}^{\left(s_{2}\right)}\left(\mathbf{k}_{1}\right) \\
& \times\left\langle\phi_{\mu}^{s_{1}}\left|\mathbf{V}_{\mathrm{SO}}\right| \phi_{\nu}^{s_{2}}\right\rangle \delta_{\mathbf{k}_{1}, \mathbf{k}_{2}} .
\end{aligned}
$$

With the result of Eq. (12) at hand we can now come back to the expression for the SO matrix elements written over the MLWFs computed in absence of spin-orbit [see Eq. (5)]. In the case of the SIESTA basis set this now reads

$$
\begin{aligned}
\left\langle w_{m \mathbf{R}_{1}}^{s_{1}}\left|\mathbf{V}_{\mathrm{SO}}\right| w_{n \mathbf{R}_{2}}^{s_{2}}\right\rangle= & \frac{1}{N} \sum_{p, q, \mu, \nu} \sum_{\mathbf{k}} C_{\mu p}^{* s_{1}}(\mathbf{k}) C_{\nu q}^{s_{2}}(\mathbf{k}) U_{p m}^{*\left(s_{1}\right)}(\mathbf{k}) \\
& \times U_{q n}^{s_{2}}(\mathbf{k}) e^{i \mathbf{k} \cdot\left(\mathbf{R}_{1}-\mathbf{R}_{2}\right)}\left\langle\phi_{\mu}^{s_{1}}\left|\mathbf{V}_{\mathrm{SO}}\right| \phi_{\nu}^{s_{2}}\right\rangle
\end{aligned}
$$


Finally, we go back to the continuous representation $(N \rightarrow$ $\infty)$, where the sum over $\mathbf{k}$ is replaced by an integral over the first Brillouin zone

$$
\begin{aligned}
\left\langle w_{m \mathbf{R}_{1}}^{s_{1}}\left|\mathbf{V}_{\mathrm{SO}}\right| w_{n \mathbf{R}_{2}}^{s_{2}}\right\rangle= & \frac{V}{(2 \pi)^{3}} \sum_{p, q, \mu, v} \int_{\mathrm{BZ}} C_{\mu p}^{* s_{1}}(\mathbf{k}) C_{\nu q}^{s_{2}}(\mathbf{k}) U_{p m}^{* s_{1}}(\mathbf{k}) \\
& \times U_{q n}^{s_{2}}(\mathbf{k}) e^{i \mathbf{k} \cdot\left(\mathbf{R}_{1}-\mathbf{R}_{2}\right)}\left\langle\phi_{\mu}^{s_{1}}\left|\mathbf{V}_{\mathrm{SO}}\right| \phi_{\nu}^{s_{2}}\right\rangle d \mathbf{k} .
\end{aligned}
$$

To summarize, our strategy consists in simply evaluating the SO matrix elements over the basis set of the MLWFs constructed in the absence of SO interaction. These are by definition spin degenerate and they are in general easy to compute since associated with well-separated bands. Our procedure thus avoids to run the minimization algorithm necessary to fix the Wannier's gauge over the SO-split bands, which in the case of OSCs have tiny splits. Our method is exact in the case the MLWFs form a complete set describing a particular bands manifold. In other circumstances they constitute a good approximation, as long as the SO interaction is weak, namely when it does not change significantly the spatial shape of the Wannier functions. However, for a material with strong SO coupling (e.g., Pb), if the MLWFs under consideration do not span the entire Bloch states manifold, then the SO-split eigenvalues calculated with our method will not match those obtained directly with the first-principles calculation.

\section{Workflow}

The following procedure is adopted when calculating the SO-split band structures from the MLWFs Hamiltonian. The results are then compared to the band structure obtained directly from SIESTA including SO interaction.

(1) We first run a self-consistent noncollinear spin-DFT SIESTA calculation and obtain the band structure.

(2) From the density matrix obtained at step (1), we run a non-self-consistent single-step SIESTA calculation including SO coupling. This gives us the matrix elements $\left\langle\phi_{\mu}^{s_{1}}\left|\mathbf{V}_{\text {SO }}\right| \phi_{v}^{s_{2}}\right\rangle$. The band structure obtained in this calculation (from now on this is called the SO-DFT band structure) will be then compared with that obtained over the MLWFs. Note that we do not perform the SIESTA DFT calculation including spinorbit interaction in a self-consistent way. This is because the SO interaction changes little the density matrix so that such calculation is often not necessary. Furthermore, as we cannot run the MLWF calculation in a self-consistent way over the SO interaction, considering non-self-consistent $\mathrm{SO}$ band structure at the SIESTA level allows us to compare electronic structures arising from identical charge densities.

(3) Since the current version of WANNIER90 implemented for SIESTA works only with collinear spins, we run a regular self-consistent spin-polarized SIESTA calculation. This gives us the coefficients $C_{\mu n}^{s}(\mathbf{k})$, which are spin degenerate for a nonmagnetic material $C_{\mu n}^{\uparrow}(\mathbf{k})=C_{\mu n}^{\downarrow}(\mathbf{k})$.

(4) We run a WANNIER90 calculation to construct the MLWFs associated with the band structure computed at point (3). This returns us the unitary matrix $U_{p m}^{s}(\mathbf{k})$, the Hamiltonian matrix elements $\left\langle w_{m \mathbf{R}_{1}}^{s_{1}}\left|\mathbf{H}_{0}\right| w_{n \mathbf{R}_{2}}^{s_{2}}\right\rangle\left(\mathbf{H}_{0}\right.$ is the

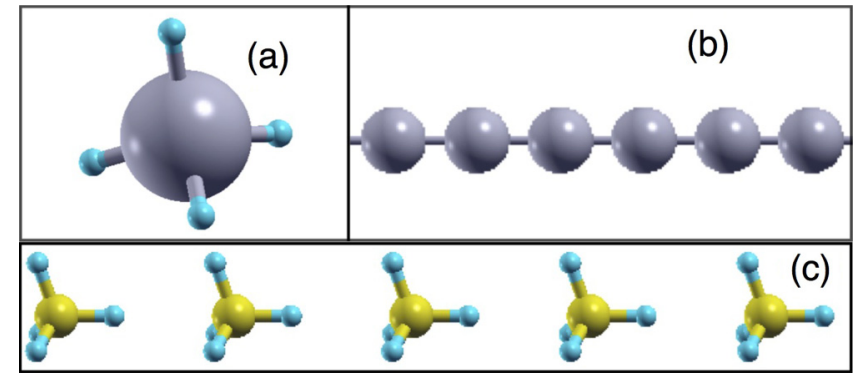

FIG. 1. Atomic structure of (a) a plumbane molecule, (b) a chain of lead atoms, and (c) a chain of methane molecules. We have also calculated the electronic structure of a chain of $\mathrm{C}$ atoms, which is essentially identical to that presented in (b). Color code: $\mathrm{Pb}=$ gray, $\mathrm{H}=$ light blue, $\mathrm{C}=$ yellow.

Kohn-Sham Hamiltonian in absence of SO interaction), and the phase factors $e^{i \mathbf{k} \cdot \mathbf{R}}$ [36]. For a nonmagnetic material the matrix elements of $\mathbf{H}_{0}$ satisfy the relation $\left\langle w_{m \mathbf{R}_{\mathbf{1}}}^{s_{1}}\left|\mathbf{H}_{0}\right| w_{n \mathbf{R}_{2}}^{s_{2}}\right\rangle=$ $\left\langle w_{m \mathbf{R}_{1}}\left|\mathbf{H}_{0}\right| w_{n \mathbf{R}_{2}}\right\rangle \delta_{s_{1}, s_{2}}$.

(5) From $\left\langle\phi_{\mu}^{s_{1}}\left|\mathbf{V}_{\mathrm{SO}}\right| \phi_{\nu}^{s_{2}}\right\rangle$ and the $C_{\mu n}^{s}(\mathbf{k})$ 's we calculate the matrix elements $\left\langle\psi_{p \mathbf{k}}^{s_{1}}\left|\mathbf{V}_{\text {SO }}\right| \psi_{q \mathbf{k}}^{s_{2}}\right\rangle$ by using Eq. (12).

(6) Next we transform the SO matrix elements constructed over the Bloch functions $\left\langle\psi_{p \mathbf{k}}^{s_{1}}\left|\mathbf{V}_{\text {SO }}\right| \psi_{q \mathbf{k}}^{s_{2}}\right\rangle$ into their Wannier counterparts $\left\langle w_{m \mathbf{R}_{1}}^{s_{1}}\left|\mathbf{V}_{\text {SO }}\right| w_{n \mathbf{R}_{2}}^{s_{2}}\right\rangle$, by using Eq. (14).

(7) The final complete Wannier Hamiltonian now reads

$$
\left\langle w_{m \mathbf{R}_{1}}^{s_{1}}|\mathbf{H}| w_{n \mathbf{R}_{2}}^{s_{2}}\right\rangle=\left\langle w_{m \mathbf{R}_{1}}^{s_{1}}\left|\mathbf{H}_{0}+\mathbf{V}_{\text {SO }}\right| w_{n \mathbf{R}_{2}}^{s_{2}}\right\rangle,
$$

and the associated band structure can be directly compared with that computed at point (2) directly from SIESTA.

\section{RESULTS AND DISCUSSION}

We now present our results, which are discussed in the light of the theory just described.

\section{A. Plumbane molecule}

We start our analysis by calculating the SO matrix elements and then the energy eigenvalues of a plumbane, $\mathrm{PbH}_{4}$, molecule [see Fig. 1(a)]. Due to the presence of lead, the molecular eigenstates change significantly when the SO interaction is switched on. For this nonperiodic system the key relations in Eqs. (12) and (5) reduce to

$$
\left\langle\psi_{p}^{s_{1}}\left|\mathbf{V}_{\mathrm{SO}}\right| \psi_{q}^{s_{2}}\right\rangle=\sum_{\mu, v} C_{\mu p}^{* s_{1}} C_{\nu q}^{s_{2}}\left\langle\phi_{\mu}^{s_{1}}\left|\mathbf{V}_{\mathrm{SO}}\right| \phi_{\nu}^{s_{2}}\right\rangle
$$

and

$$
\left\langle w_{m}^{s_{1}}\left|\mathbf{V}_{\mathrm{SO}}\right| w_{n}^{s_{2}}\right\rangle=\sum_{p, q} U_{p m}^{* s_{1}} U_{q n}^{s_{2}}\left\langle\psi_{p}^{s_{1}}\left|\mathbf{V}_{\mathrm{SO}}\right| \psi_{q}^{s_{2}}\right\rangle,
$$

respectively, where now the vectors $\psi_{n}^{s}$ are simply the eigenvectors with quantum number $n$ and spin $s$.

In Table I we report the first ten energy eigenvalues of plumbane, calculated either with or without SO coupling. These have been computed within the LDA (local density approximation) and a double-zeta polarized basis set. The table compares results obtained with our MLWFs procedure to those computed with SO-DFT by SIESTA. Clearly in this case of a heavy ion the SO coupling changes the eigenvalues 
TABLE I. The ten lowest energy eigenvalues of a plumbane molecule calculated with (SO) and without (non-SO) spin-orbit interaction. The first and third columns correspond to the SO-DFT SIESTA calculation, while the second and the fourth to the MLWFs one. The $M R A D$ for both cases is reported in the last row.

\begin{tabular}{lcccc}
\hline \hline \multicolumn{2}{c}{ Non-SO } & & \multicolumn{2}{c}{ SO } \\
\cline { 5 - 5 } SIESTA & MLWF & & SIESTA & MLWF \\
\hline-33.93534 & -33.93521 & & -33.93532 & -33.93521 \\
-33.93530 & -33.93521 & & -33.93528 & -33.93521 \\
-13.02511 & -13.02507 & & -14.69573 & -14.69568 \\
-13.02511 & -13.02507 & & -14.69573 & -14.69568 \\
-13.02510 & -13.02506 & & -12.64301 & -12.64298 \\
-13.02509 & -13.02506 & & -12.64301 & -12.64298 \\
-13.02320 & -13.02315 & & -12.64166 & -12.64162 \\
-13.02318 & -13.02315 & & -12.64165 & -12.64162 \\
-5.75256 & -5.75251 & & -5.75255 & -5.75251 \\
-5.75245 & -5.75251 & & -5.75245 & -5.75251 \\
$M R A D=4.320 \times 10^{-6}$ & & MRAD $=3.998 \times 10^{-6}$ \\
\hline \hline
\end{tabular}

appreciably, in particular in the spectral region around -13 $\mathrm{eV}$. Such change is well captured by our Wannier calculation, which returns energy levels in close proximity to those computed with SO-DFT by SIESTA. In order to estimate the error introduced by our method, we calculate the mean relative absolute difference (MRAD), which we define as $\frac{1}{N} \sum \frac{\left|\epsilon_{i}^{s}-\epsilon_{i}^{w}\right|}{\left|\epsilon_{i}^{s}\right|}$ for a set of $N$ eigenvalues $(i=1, \ldots, N)$, where $\epsilon_{i}^{s}$ and $\epsilon_{i}^{w}$ are the $i$ th eigenvalues calculated from SIESTA and the MLWFs, respectively. Notably the $M R A D$ is rather small both in the SO-free case and when the SO interaction is included. Most importantly, we can report that our procedure to evaluate the SO matrix elements over the MLWFs basis clearly does not introduce any additional error.

Before discussing some of the properties of the SO matrix elements associated with this particular case of a finite molecule, we wish to make a quick remark on the Wannier procedure adopted here. The eigenvalues reported in Table I are the ten with the lowest energies. However, in order to construct the MLWFs we have considered all the states of the calculated Kohn-Sham spectrum. This means that if our SIESTA basis set describes $\mathrm{PbH}_{4}$ with $N$ distinct atomic orbitals, then the MLWFs constructed are $2 N$ (the factor 2 accounts for the spin degeneracy). In this case the original local orbital basis set and the constructed MLWFs span the same Hilbert space and the mapping is exact, whether or not the SO interaction is considered.

In most cases, however, one wants to construct the MLWFs by using only a subset of the spectrum, for instance the first $N^{\prime}$ eigenstates. Since in general the SO interaction mixes all states, there will be SO matrix elements between the selected $N^{\prime}$ states and the remaining $N-N^{\prime}$. This means that a MLWF basis constructed only from the first $N^{\prime}$ eigenstates will not be able to provide an accurate description of the SO-split spectrum. Importantly, one in general may expect that the SO interaction matrix elements between different Kohn-Sham orbitals $\left\langle\psi_{p}^{s_{1}}\left|\mathbf{V}_{\mathrm{SO}}\right| \psi_{q}^{s_{2}}\right\rangle$ are smaller than those calculated at the same orbital $\left\langle\psi_{n}^{s_{1}}\left|\mathbf{V}_{\text {SO }}\right| \psi_{n}^{s_{2}}\right\rangle$. This is because of the short range of the SO interaction and the fact that the Kohn-Sham eigenstates are orthonormal. In the case of light elements, i.e., for a weak SO potential, one may completely neglect the off-diagonal SO matrix elements. This means that the SO spectrum constructed with the MLWFs associated with the first $N^{\prime}$ eigenstates will be approximately equal to the first $N^{\prime}$ eigenvalues of the MLWFs Hamiltonian constructed over the entire $N$-dimensional spectrum. Such property is particularly relevant for OSCs, for which the SO interaction is weak.

We now move to discuss a general property of the MLWF SO matrix elements, namely the relations $\left\langle w_{m}^{s}\left|\mathbf{V}_{\text {SO }}\right| w_{m}^{s}\right\rangle=0$ and $\operatorname{Re}\left[\left\langle w_{m}^{s}\left|\mathbf{V}_{\text {SO }}\right| w_{n}^{s}\right\rangle\right]=0$. This means that the SO matrix elements for the same spin and the same Wannier function vanish, while those for the same spin and different Wannier functions are purely imaginary. This property can be understood from the following argument. The SO coupling operator is $\mathbf{V}_{\text {SO }}=\sum_{\mathbf{R}_{j}} V_{\mathbf{R}_{j}} \mathbf{L}_{\mathbf{R}_{j}} \cdot \mathbf{S}$, where $V_{\mathbf{R}_{j}}$ is a scalar potential independent of spin, and $\mathbf{L}_{\mathbf{R}_{j}}$ is the angular momentum operator corresponding to the central potential of the atom at position $\mathbf{R}_{j}$. Here $\mathbf{S}$ is the spin operator and the sum runs over all the atoms. By now expanding $\mathbf{S}$ in terms of the Pauli spin matrices one can see that for any vector $\left|\gamma_{i}^{s}\right\rangle=\left|\gamma_{i}\right\rangle \otimes|s\rangle$, which can be written as a tensor product of a spin-independent part $\left|\gamma_{i}\right\rangle$ and a spinor $|s\rangle$, the following equality holds:

$$
\begin{aligned}
\left\langle\gamma_{m}^{s_{1}}|\mathbf{L} \cdot \mathbf{S}| \gamma_{n}^{s_{2}}\right\rangle= & \frac{1}{2}\left[\left\langle\gamma_{m}\left|\hat{L}_{z}\right| \gamma_{n}\right\rangle \delta_{s_{1} \uparrow} \delta_{s_{2} \uparrow}\right. \\
& +\left\langle\gamma_{m}\left|\hat{L}_{-}\right| \gamma_{n}\right\rangle \delta_{s_{1} \uparrow} \delta_{s_{2} \downarrow} \\
& +\left\langle\gamma_{m}\left|\hat{L}_{+}\right| \gamma_{n}\right\rangle \delta_{s_{1} \downarrow} \delta_{s_{2} \uparrow} \\
& \left.+\left\langle\gamma_{m}\left|-\hat{L}_{z}\right| \gamma_{n}\right\rangle \delta_{s_{1} \downarrow} \delta_{s_{2} \downarrow}\right] .
\end{aligned}
$$

Equation (18) can then be applied to both the Kohn-Sham eigenstates and the MLWFs, since they are both written as $\left|\gamma_{i}^{s}\right\rangle=\left|\gamma_{i}\right\rangle \otimes|s\rangle$.

Now, the atomic orbitals used by SIESTA have the following form:

$$
\left|\phi_{i}\right\rangle=\left|R_{n_{i}, l_{i}}\right\rangle \otimes\left|l_{i}, M_{i}\right\rangle
$$

where $\left|R_{n, l}\right\rangle$ is a radial numerical function, while the angular dependence is described by the real spherical harmonic $|l, M\rangle$ [37]. It can be proved that the real spherical harmonics follow the relation

$$
\left\langle l, M_{i}\left|\hat{L}_{z}\right| l, M_{j}\right\rangle=-i M_{i} \delta_{M_{i}, M_{j}}
$$

Since any Kohn-Sham eigenstate $\left|\psi_{p}^{s_{1}}\right\rangle$ can be written as $\left|\phi_{i}\right\rangle \otimes\left|s_{1}\right\rangle$, Eq. (18) implies that only the terms in $\hat{L}_{z}$ (or $-\hat{L}_{z}$ ) contribute to the matrix element between same spins $\left\langle\psi_{p}^{s_{1}}|\mathbf{L} \cdot \mathbf{S}| \psi_{p}^{s_{1}}\right\rangle$. Equation (20) together with the fact that the Kohn-Sham eigenstates are real for a finite molecule further establishes that $\operatorname{Re}\left[\left\langle\psi_{p}\left|\hat{L}_{z}\right| \psi_{q}\right\rangle\right]=0$. As a consequence $\left\langle\psi_{m}\left|\hat{L}_{z}\right| \psi_{m}\right\rangle=0$. Finally, by keeping in mind that the unitary matrix elements transforming the Kohn-Sham eigenstates into MLWFs are real for a molecule, we have also

$$
\begin{aligned}
\left\langle w_{m}^{s_{1}}|\mathbf{L} \cdot \mathbf{S}| w_{n}^{s_{1}}\right\rangle & = \pm\left\langle w_{m}\left|\hat{L}_{z}\right| w_{n}\right\rangle \\
& =\sum_{p \neq q} U_{p m} U_{q n}\left\langle\psi_{p}\left|\hat{L}_{z}\right| \psi_{q}\right\rangle,
\end{aligned}
$$




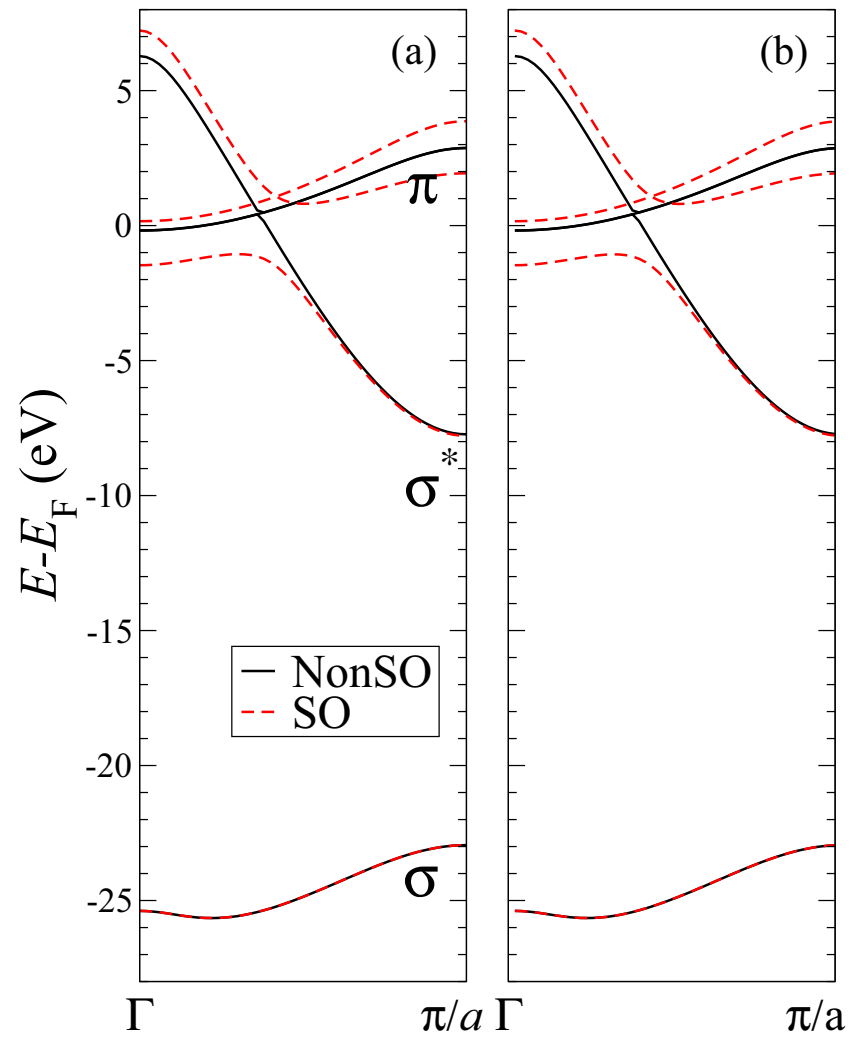

FIG. 2. Band structure of a $1 \mathrm{D} \mathrm{Pb}$ chain calculated (a) with SIESTA and (b) by diagonalizing the Hamiltonian matrix constructed over the MLWFs. Black and red lines are for the bands obtained without and with SO coupling, respectively. The $\sigma, \sigma^{*}$, and $\pi$ bands are identified in the picture.

which has to be imaginary. Thus we have $\operatorname{Re}\left\langle w_{m}^{s_{1}}\left|\mathbf{V}_{\mathrm{SO}}\right| w_{n}^{s_{1}}\right\rangle=$ 0 and $\left\langle w_{m}^{s_{1}}\left|\mathbf{V}_{\text {SO }}\right| w_{m}^{s_{1}}\right\rangle=0$ since $\mathbf{V}_{\text {SO }}$ must have real expectation values.

\section{B. Lead chain}

Next we move to calculating the SO matrix elements for a periodic structure. In particular we look at a 1D chain of $\mathrm{Pb}$ atoms with a unit cell length of $2.55 \AA$, which is the DFT equilibrium lattice constant obtained with the LDA. Note that free-standing monodimensional $\mathrm{Pb}$ chains have been never reported in literature, although there are studies of low-dimensional $\mathrm{Pb}$ structures encapsulated into zeolites [38]. Here, however, we do not seek at describing a real compound, but we rather take the $1 \mathrm{D} \mathrm{Pb}$ monoatomic chain as a test-bench structure to apply our method to a periodic structure with a large SO coupling. Also in this case we have constructed the MLWFs by taking the entire bands manifold and not a subset of it. For the DFT calculations we have considered a simple $s$ and $p$ single-zeta basis set, which, in absence of SO interaction, yields three bands with one of them being doubly degenerate [see Fig. 2(a)]. The doubly degenerate relatively flat band just cuts across the Fermi energy $E_{\mathrm{F}}$ and it is composed of the $p_{y}$ and $p_{z}$ orbitals orthogonal to the chain axis ( $\pi$ band). The other two bands are $s p$ hybrid ( $\sigma$ bands). The lowest one at about $25 \mathrm{eV}$ below $E_{\mathrm{F}}$ has mainly $s$ character ( $\sigma$ band), while the other mainly $p_{x}\left(\sigma^{*}\right.$ band).
Spin-orbit coupling lifts the degeneracy of the $p$-type band manifold, which is now composed of three distinct bands. In particular the degeneracy is lifted only in the $\pi$ band at the edge of the 1D Brillouin zone, while it also involves the $\sigma$ one close to the $\Gamma$ point (after the band crossing). When the same band structure is calculated from the MLWFs we obtain the plot of Fig. 2(b). This is almost identical to that calculated with SO-DFT demonstrating the accuracy of our method also for periodic system.

It must be noted that for a periodic structure the Bloch state expansion coefficients $C_{\mu p}(\mathbf{k})$ and the elements of the unitary matrix $U$ are complex and consequently the diagonal elements of $\mathbf{V}_{\text {So }}$ with respect to Wannier functions are not zero in general. However, as expected $\left\langle w_{m \mathbf{R}}^{s_{1}}\left|\mathbf{V}_{\text {SO }}\right| w_{n \mathbf{R}^{\prime}}^{s_{2}}\right\rangle$ tends to vanish as the separation $\left|\mathbf{R}-\mathbf{R}^{\prime}\right|$ increases. Furthermore, it is clear from Eq. (18) that the SO matrix elements for Wannier functions should obey the spin-box anti-Hermitian relation

$$
\left\langle w_{m \mathbf{R}}^{s_{1}}\left|\mathbf{V}_{\mathrm{SO}}\right| w_{n \mathbf{R}^{\prime}}^{s_{2}}\right\rangle=-\left\langle w_{m \mathbf{R}}^{s_{2}}\left|\mathbf{V}_{\mathrm{SO}}\right| w_{n \mathbf{R}^{\prime}}^{s_{1}}\right\rangle^{*} .
$$
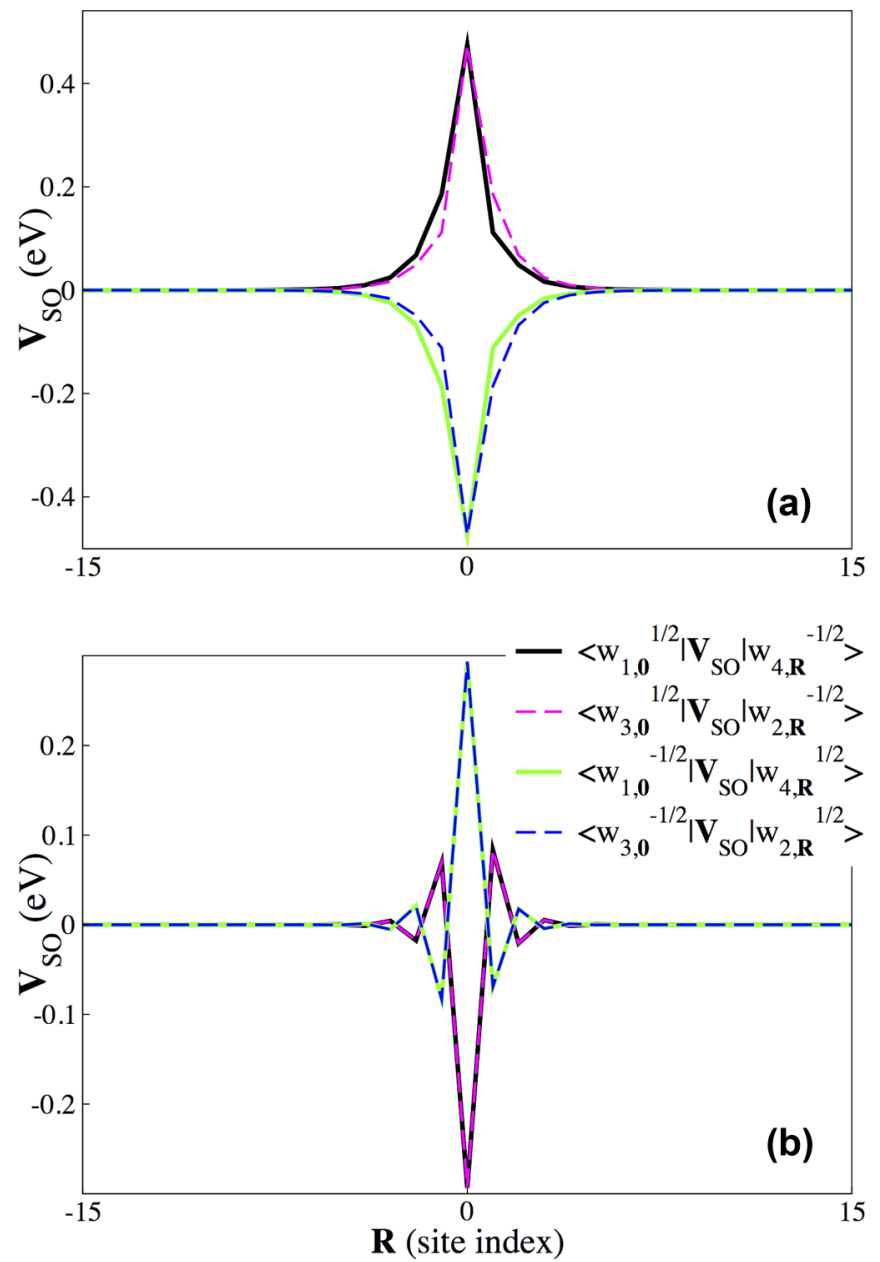

FIG. 3. The SO matrix elements of a chain of lead atoms calculated with respect to some representative Wannier functions and plotted as a function of the site index, i.e., of the distance between the Wannier function. (a) and (b) The real and imaginary components, respectively. 
These two properties can be appreciated in Fig. 3, where we plot the real [Fig. 3(a)] and imaginary [Fig. 3(b)] part of $\left\langle w_{m \mathbf{0}}^{s_{1}}\left|\mathbf{V}_{\text {SO }}\right| w_{n \mathbf{R}}^{s_{2}}\right\rangle$ for some representative band combinations $m$ and $n$ as a function of $\mathbf{R}$.

\section{Carbon chain}

Next we look at the case of a 1D monoatomic carbon chain with a LDA-relaxed interatomic distance of $\sim 1.3 \AA$. This has the same structure and electron count of the $\mathrm{Pb}$ chain, and the only difference concerns the fact that the SO coupling in $\mathrm{C}$ is much smaller then that in $\mathrm{Pb}$. In this situation we expect that an accurate SO-split band structure can be obtained even when the MLWFs are constructed only for a limited number of bands and not for the entire band manifold as in the case of $\mathrm{Pb}$. This time the DFT band structure is calculated at the LDA level over a double-zeta polarized (DZP) SIESTA basis set, comprising 13 atomic orbitals per unit cell. In contrast, the MLWFs are constructed only from the first four bands, which are well isolated in energy from the rest and again describe the $s p$ bands with $\sigma$ and $\pi$ symmetry. Since the SO interaction in carbon is small (the band split is of the order of a few meV) it is impossible to visualize the effects of the SO interaction in a standard band plot as that in Fig. 2. Hence, in Fig. 4 we plot the difference between the band structure calculated in the presence and in the absence of SO coupling. In particular we compare the bands calculated with SO-DFT by SIESTA (left-hand side panels in Fig. 4), with those obtained with the MLWFs scheme described here (right-hand side panels in Fig. 4). In the figure we have labeled the bands in order of increasing energy and neglecting the spin degeneracy. Thus, for instance, the $\psi_{1}$ and $\psi_{2}$ bands correspond to the two lowest $\sigma$ spin subbands (note that the band structure of the linear carbon chain is qualitatively identical to that of the $\mathrm{Pb}$ one and we can use Fig. 2 to identify the various bands).

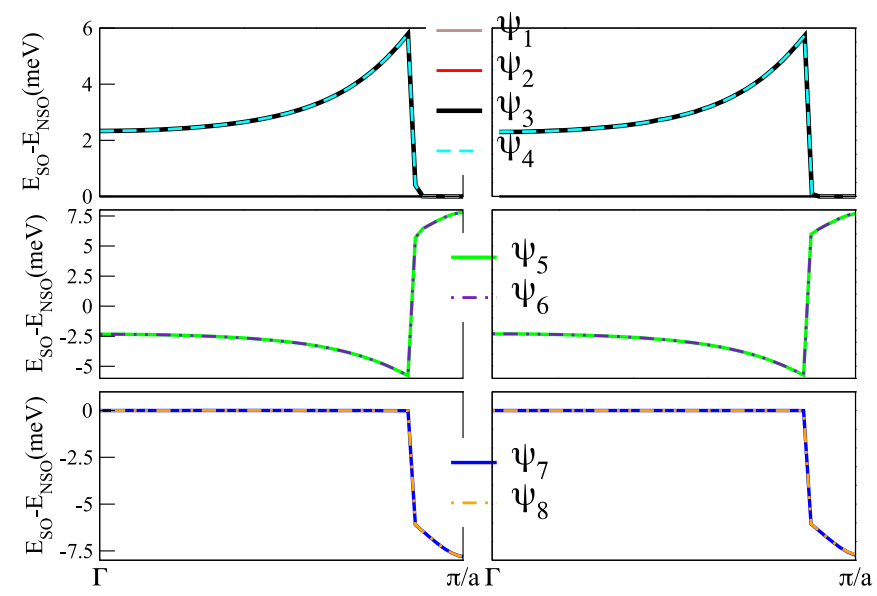

FIG. 4. Difference $E_{\mathrm{SO}}-E_{\mathrm{NSO}}$ between the band structure of chain of carbon atoms calculated with $E_{\mathrm{SO}}$ and without $E_{\mathrm{NSO}}$, considering SO interaction. The bands are labeled in increasing energy order without taking into account spin degeneracy. For instance the bands $\psi_{1}$ and $\psi_{2}$ are the two spin subbands corresponding to the $\sigma$ band (see Fig. 2 for notation). The left-hand side panels show results for the SO-DFT calculations performed with SIESTA, while the right-hand side one, those obtained from the MLWFs.
We note that the lowest $\sigma$ bands, defined as $\psi_{1}$ and $\psi_{2}$, do not split at all due to the SO interaction, exactly as in the case of $\mathrm{Pb}$. This contrasts the behavior of both the $\pi\left(\psi_{3}\right.$ through $\left.\psi_{6}\right)$ and $\sigma^{*}\left(\psi_{7}\right.$ and $\left.\psi_{8}\right)$ bands, which instead are modified by the SO interaction. Notably the changes in energy of the eigenvalues is never larger then $8 \mathrm{meV}$ and it is perfectly reproduced by our MLWFs representation. This demonstrates that truncating the bands selected for constructing the MLWFs is a possible procedure for materials where SO coupling is weak. However, we should note that the truncation still needs to be carefully chosen. Here for instance we have considered all the $2 s$ and $2 p$ bands and neglected those with either higher principal quantum number (e.g., $3 s$ and $3 p$ ) or higher angular momentum (e.g., bands with $d$ symmetry originating from the $p$-polarized SIESTA basis), which appear at much higher energies. Truncations, where one considers only a particular orbital of a given shell (say the $p_{z}$ orbital in an $n p$ shell), need to be carefully assessed since it is unlikely that a clear energy separation between the bands takes place.

\section{Methane chain}

As a first basic prototype of 1D organic molecular crystal we perform calculations for a periodic chain of methane molecules. We use a double-zeta polarized basis set and a LDA-relaxed unit cell length of $3.45 \AA$ (the cell contains only one molecule). Similarly to the previous case, the MLWFs are constructed over only the lowest four bands (eight when considering the spin degeneracy). When compared to the bands of the carbon chain, those of methane are much narrower. This is expected, since the bonding between the different molecules is small. In Fig. 5 we plot the difference between

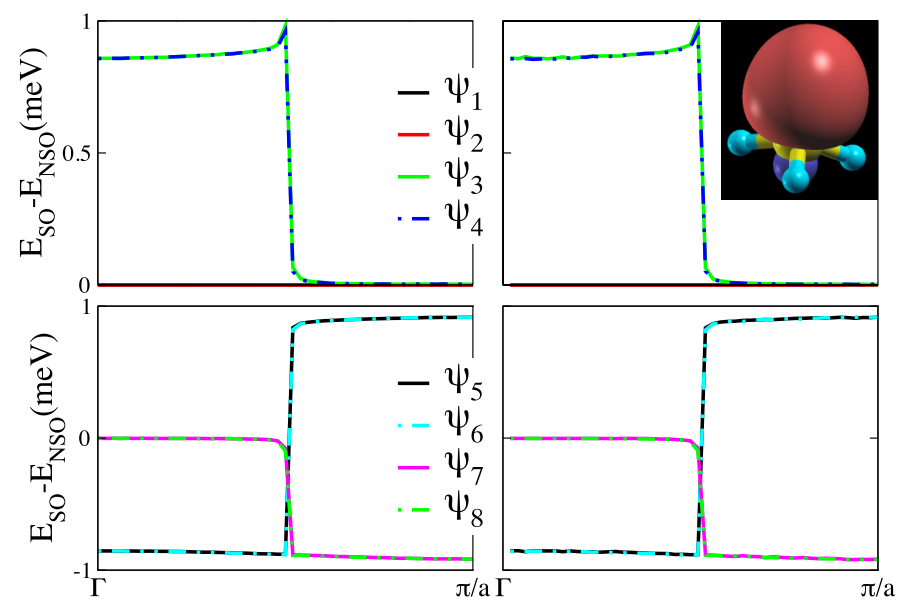

FIG. 5. Difference $E_{\mathrm{SO}}-E_{\mathrm{NSO}}$ between the band structure of chain of methane molecules calculated with $E_{\mathrm{SO}}$ and without $E_{\mathrm{NSO}}$ considering SO interaction. The bands are labeled in increasing energy order without taking into account spin degeneracy. The left-hand side panels show results for the SO-DFT calculations performed with SIESTA, while the right-hand side one, those obtained from the MLWFs. The inset shows an isovalue plot of one of the four MLWFs with the red and blue surfaces denoting positive and negative isovalues, respectively. All the MLWFs have similar structure and they resemble those of the isolated methane molecule because of the small intermolecular chemical bonding owing to the large separation. 
the eigenvalues (1D band structure) calculated with $E_{\mathrm{SO}}$ and without $E_{\mathrm{NSO}}$ including SO interaction.

When SO interaction is included the spin degeneracy is broken and one has now eight bands. These are labeled as $\psi_{m}$ in Fig. 5 in increasing energy order. Again we find no SO split for the lowermost band and then a split, which is significantly smaller than that found in the case of the $\mathrm{C}$ chain. This is likely to originate from the crystal field of the $\mathrm{C}$ atoms in $\mathrm{CH}_{4}$, which is different from that in the $\mathrm{C}$ chain (the $\mathrm{C}$ - $\mathrm{C}$ distance is different and there are additional C-H bonds). Again, as in the previous case, we find that our MLWFs procedure perfectly reproduces the SO-DFT band structure, indicating that in this case of weak SO interaction band truncation does not introduce any significant error.

\section{E. Triarylamine chain}

Finally we perform calculations for a real system, namely for triarylamine-based molecular nanowires. These can be experimentally grown through a photo-self-assembly process from the liquid phase [39], and have been subject of numerous experimental and theoretical studies [34,40]. In general, triarylamines can be used as materials for organic light emitting diodes, while their nanowire form appears to possess good transport and spin properties, making it a good platform for organic spintronics [41]. Triarylamine-based molecular nanowires self-assemble only when particular radicals are attached to the main triarylamine backbone and here we

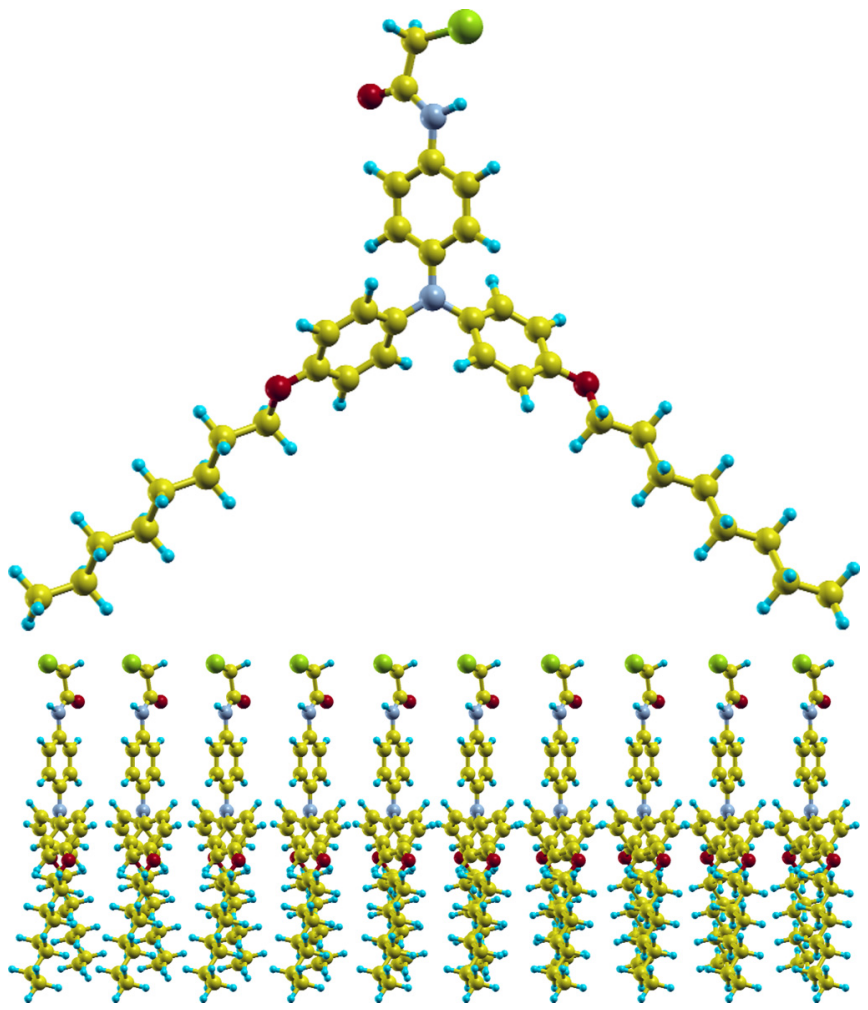

FIG. 6. Structure of the triarylamine molecule (upper picture) and of the triarylamine-based nanowire investigated here. The radicals associated with the triarylamine derivative are $\mathrm{C}_{8} \mathrm{H}_{17}, \mathrm{H}$, and $\mathrm{Cl}$, respectively. Color code: $\mathrm{C}=$ yellow, $\mathrm{H}=$ light blue, $\mathrm{O}=$ red, $\mathrm{N}=$ gray, $\mathrm{Cl}=$ green.

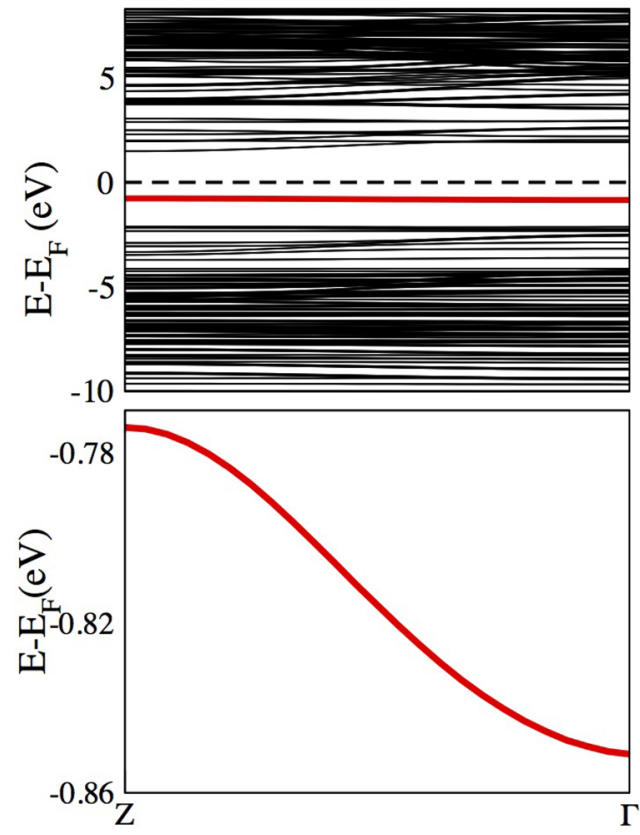

FIG. 7. Band structure of the $1 \mathrm{D}$ triarylamine-based nanowire constructed with the precursor 1 of Ref. [39]. This is plotted over the $1 \mathrm{D}$ Brillouin zone ( $\mathrm{Z}=\pi / a$ with $a$ the lattice parameter). The Fermi level is marked with a dashed black line and it is placed just above the HOMO-derived valence band (in red). The lower panel is a magnification of the valence band. Note the bandwidth of about $100 \mathrm{meV}$ and the fact that the band has a cosine shape, fingerprint of a single-orbital nearest-neighbor tight-binding-like interaction. Only the HOMO band is considered when constructing the MLWFs.

consider the case of $\mathrm{C}_{8} \mathrm{H}_{17}, \mathrm{H}$, and $\mathrm{Cl}$ radicals, corresponding to the precursor 1 of Ref. [39] (see upper panel in Fig. 6). The nanowire then arranges in such a way to have the central $\mathrm{N}$ atoms aligned along the wire axis (see Fig. 6).

In general self-assembled triarylamine-based molecular nanowires appear slightly $p$ doped so that charge transport takes place in the HOMO-derived band. This is well isolated from the rest of the valence manifold and has a bandwidth of about $100 \mathrm{meV}$ (see Fig. 7 for the band structure). Such a band is almost entirely localized on the $p_{z}$ orbital of the central $\mathrm{N}$ atoms ( $p_{z}$ is along the wire axis), a feature that has allowed us to construct a $p_{z}-s p^{2}$ model with the spin-orbit strength extracted from that of an equivalent monoatomic $\mathrm{N}$ chain. The model was then used to calculate the temperature-dependent spin-diffusion length of such nanowires [42]. Here we wish to use our MLWFs method to extract the SO matrix elements of triarylamine-based molecular nanowires in their own chemical environment, i.e., without approximating the backbone with a $\mathrm{N}$ atomic chain.

For this system we use a 1D lattice with LDA-optimized lattice spacing of $4.8 \AA$ and run the DFT calculations with double-zeta polarized basis and the LDA functional. The MLWFs are constructed by using only the HOMO-derived valence band, i.e., we have a single spin-degenerate Wannier orbital. We can then drop the band index and write the SO 


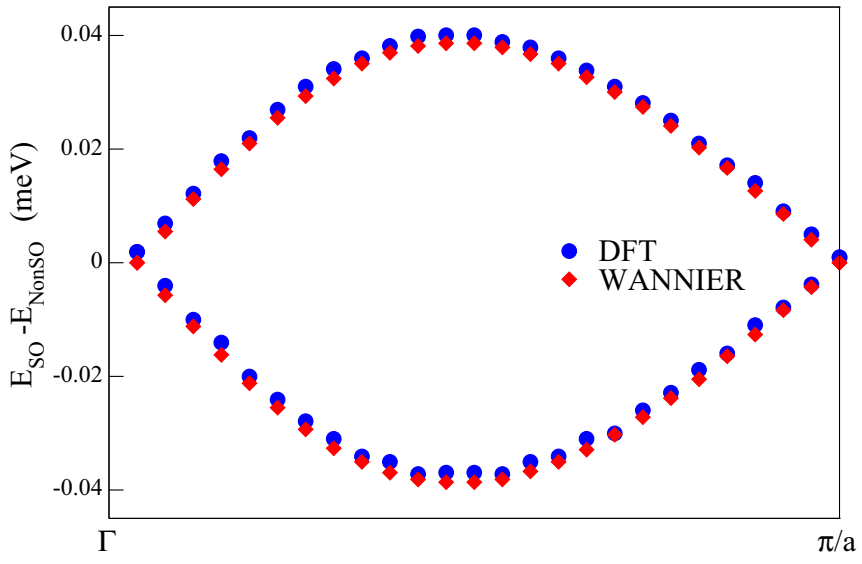

FIG. 8. Plot of ( $\left.E_{\mathrm{SO}}-E_{\mathrm{NSO}}\right)$ as a function of $\mathbf{k}$ in arbitrary unit over a Brillouin zone for the highest occupied band of a 1D chain of triarylamine derivatives. The blue and the red points correspond to calculations with SIESTA and WANNIER90, respectively.

matrix elements as

$$
\begin{aligned}
\left\langle w_{\mathbf{0}}^{s_{1}}\left|\mathbf{V}_{\mathrm{SO}}\right| w_{\mathbf{R}}^{s_{2}}\right\rangle= & \frac{V}{(2 \pi)^{3}} \int d \mathbf{k} U^{*}(\mathbf{k}) U(\mathbf{k}) e^{-i \mathbf{k} \cdot \mathbf{R}} \\
& \times\left\langle\psi_{\mathbf{k}}^{s_{1}}\left|\mathbf{V}_{\mathrm{SO}}\right| \psi_{\mathbf{k}}^{s_{2}}\right\rangle \\
= & \frac{V}{(2 \pi)^{3}} \int d \mathbf{k} e^{-i \mathbf{k} \cdot \mathbf{R}}\left\langle\psi_{\mathbf{k}}^{s_{1}}\left|\mathbf{V}_{\mathrm{SO}}\right| \psi_{\mathbf{k}}^{s_{2}}\right\rangle,
\end{aligned}
$$

or in a discrete representation of the reciprocal space

$$
\left\langle w_{\mathbf{0}}^{s_{1}}\left|\mathbf{V}_{\mathrm{SO}}\right| w_{\mathbf{R}}^{s_{2}}\right\rangle=\frac{1}{N} \sum_{\mathbf{k}} e^{-i \mathbf{k} \cdot \mathbf{R}}\left\langle\psi_{\mathbf{k}}^{s_{1}}\left|\mathbf{V}_{\mathrm{SO}}\right| \psi_{\mathbf{k}}^{s_{2}}\right\rangle,
$$

where the second equality comes from the unitarity of the gauge transformation $U(\mathbf{k})$.

In Fig. 8 we plot the difference between the band structure computed by including SO interaction and those calculated without. Notably our MLWFs band structure is almost identical to that computed directly with SO-DFT, again demonstrating both the accuracy of our method and the appropriateness of the drastic band truncation used here. In this particular case the SO band split is maximized halfway between the $\Gamma$ point and the edge of the $1 \mathrm{D}$ Brillouin zone, where it takes a value of approximately $80 \mu \mathrm{eV}$. Clearly such a split is orders of magnitude smaller than the value that one can possibly calculate by a direct construction of the MLWFs from the SO-splitted band structure. Note also that the SO split of the valence band is calculated here approximately a factor 10 smaller than that estimated previously for a $\mathrm{N}$ atomic chain [42], indicating the importance of the details of the chemical environment in these calculations.

Finally we take a closer look at the calculated SO matrix elements. As mentioned earlier, in the SIESTA on-site approximation [35] only the matrix elements calculated over orbitals centered on the same atom do not vanish. As a consequence the components $\left\langle w_{\mathbf{R}}^{s_{1}}\left|\mathbf{V}_{\text {SO }}\right| w_{\mathbf{R}^{\prime}}^{s_{2}}\right\rangle$ drop to zero as $\left|\mathbf{R}-\mathbf{R}^{\prime}\right|$ gets large. This can be clearly appreciated in Figs. 9(a) and 9(b), where we plot the SO matrix elements for same and different spins, respectively.

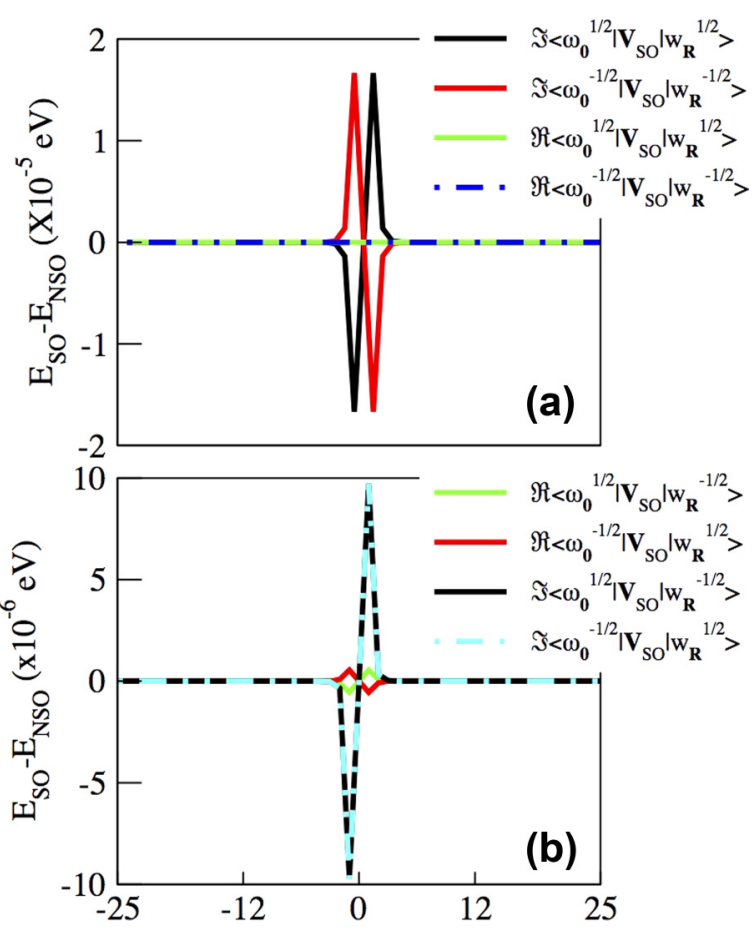

FIG. 9. SO matrix elements of a triarylamine-based nanowire calculated with respect to the Wannier functions obtained from the HOMO band. (a) and (b) Matrix elements calculated between for same and different spins, respectively.

From Fig. 9(a) we can observe that $\operatorname{Re}\left\langle w_{\mathbf{0}}^{s_{1}}\left|\mathbf{V}_{\text {SO }}\right| w_{\mathbf{R}}^{s_{1}}\right\rangle$ vanishes for all $\mathbf{R}$. This can be understood in the following way. In general any expectation value of $\mathbf{V}_{\text {SO }},\left\langle\psi_{\mathbf{k}}^{s}\left|\mathbf{V}_{\text {SO }}\right| \psi_{\mathbf{k}}^{s}\right\rangle$, has to be real. This is in fact antisymmetric with respect to $\mathbf{k}$, i.e., we have $\left\langle\psi_{\mathbf{0}+\mathbf{k}}^{s}\left|\mathbf{V}_{\text {SO }}\right| \psi_{\mathbf{0}+\mathbf{k}}^{s}\right\rangle=-\left\langle\psi_{\mathbf{0}-\mathbf{k}}^{s}\left|\mathbf{V}_{\text {SO }}\right| \psi_{\mathbf{0}-\mathbf{k}}^{s}\right\rangle$, where $\mathbf{k}=\mathbf{0}$ denotes the $\Gamma$ point of the Brillouin zone. Additionally, $e^{i \mathbf{k} \cdot \mathbf{R}}$ satisfies the relation $e^{i(\mathbf{0}+\mathbf{k}) \cdot \mathbf{R}}=\left[e^{i(\mathbf{0}-\mathbf{k}) \cdot \mathbf{R}}\right]^{*}$. Hence, by performing the $\mathbf{k}$ sum over first Brillouin zone we can write

$$
\operatorname{Re}\left\langle w_{\mathbf{0}}^{s_{1}}\left|\mathbf{V}_{\text {SO }}\right| w_{\mathbf{R}}^{s_{1}}\right\rangle=\operatorname{Re} \sum_{\mathbf{k}} e^{-i \mathbf{k} . \mathbf{R}}\left\langle\psi_{\mathbf{k}}^{s_{1}}\left|\mathbf{V}_{\text {SO }}\right| \psi_{\mathbf{k}}^{s_{1}}\right\rangle=0
$$

where $\left\langle w_{\mathbf{0}}^{s_{1}}\left|\mathbf{V}_{\text {SO }}\right| w_{\mathbf{0}}^{s_{1}}\right\rangle$ is the expectation value of $\mathbf{V}_{\text {SO }}$ and must be real. This implies

$$
\left\langle w_{\mathbf{0}}^{s_{1}}\left|\mathbf{V}_{\mathrm{SO}}\right| w_{\mathbf{0}}^{s_{1}}\right\rangle=0 .
$$

We can also see from Fig. 9(b) that for triarylamine the matrix elements $\left\langle w_{\mathbf{R}}^{s_{1}}\left|\mathbf{V}_{\text {SO }}\right| w_{\mathbf{R}}^{s_{2}}\right\rangle$ are almost zero for $s_{1} \neq s_{2}$. This follows directly from Eq. (18). In fact in the particular case of triarylamine nanowires the Wannier functions are constructed from one band only. As such, in order to have a nonzero matrix element $\left\langle w_{\mathbf{R}}^{s_{1}}\left|\mathbf{V}_{\text {SO }}\right| w_{\mathbf{R}}^{s_{2}}\right\rangle$, we must have nonzero values for $\left\langle w_{\mathbf{R}}\left|\hat{L}_{ \pm}\right| w_{\mathbf{R}}\right\rangle$. Therefore, the band under consideration must contain an appreciable mix of components of both the $|l, p\rangle$ and $|l, p+1\rangle$ complex spherical harmonics for some $l$ and $p$. As mentioned earlier, the triarylamine HOMO band is composed mostly of $p_{z} \mathrm{~N}$ orbitals. Hence, it has to be expected that the $\left\langle w_{\mathbf{R}}^{s_{1}}\left|\mathbf{V}_{\text {SO }}\right| w_{\mathbf{R}}^{s_{2}}\right\rangle$ matrix elements are small. 


\section{CONCLUSION}

We have presented an accurate method for obtaining the SO matrix elements between the MLWFs constructed in absence of SO coupling. Our procedure, implemented within the atomic-orbital-based DFT code SIESTA, allows one to avoid the construction of the Wannier functions over the SO-split band structure. In some cases, in particular for organic crystals, such splits are tiny and a direct construction is numerically impossible. The method is then put to the test for a number of materials systems, going from isolated molecules, to atomic nanowires, to $1 \mathrm{D}$ molecular crystals. When the entire band manifold is used for constructing the MLWFs the mapping between Bloch and Wannier orbitals is exact and the method can be used for both light and heavy elements. In contrast for weak spin-orbit interaction one can construct the MLWFs on a subset of the states in the band structures without any loss of accuracy. As such our scheme appears as an important tool for constructing effective spin Hamiltonians for organic materials to be used as input in a multiscale approach to their thermodynamical properties.

\section{ACKNOWLEDGMENTS}

This work is supported by the European Research Council, Quest project. Computational resources have been provided by the supercomputer facilities at the Trinity Center for High Performance Computing (TCHPC) and at the Irish Center for High End Computing (ICHEC). Additionally, the authors would like to thank Ivan Rungger and Carlo Motta for helpful discussions and Akinlolu Akande for providing the structure of the triarylamine-based nanowire.
[1] S. A. Wolf, D. D. Awschalom, R. A. Buhrman, J. M. Daughton, S. von Molnár, M. L. Roukes, A. Y. Chtchelkanova, and D. M. Treger, Science 294, 1488 (2001).

[2] S. Ornes, Proc. Natl. Acad. Sci. U.S.A. 110, 3710 (2013).

[3] B. Behin-Aein, D. Datta, S. Salahuddin, and S. Datta, Nat. Nanotechnol. 5, 266 (2010).

[4] G. Prinz and K. Hathaway, Phys. Today 48(4), 24 (1995).

[5] D. D. Awschalom and M. E. Flatté, Nat. Phys. 3, 153 (2007).

[6] I. Žutić and M. Fuhrer, Nat. Phys. 1, 85 (2005).

[7] G. Horowitz, in Organic Transistors, edited by H. Klauk (WileyVCH, Berlin, 2006).

[8] C. Joachim, J. K. Gimzewski, and A. Aviram, Nature (London) 408, 541 (2000).

[9] V. Dediu, M. Murgia, F. Matacotta, C. Taliani, and S. Barbanera, Solid State Commun. 122, 181 (2002).

[10] Z. H. Xiong, D. Wu, Z. Valy Vardeny, and J. Shi, Nature (London) 427, 821 (2004).

[11] S. Sanvito, Chem. Soc. Rev. 40, 3336 (2011).

[12] C. K. Chiang, C. R. Fincher, Y. W. Park, A. J. Heeger, H. Shirakawa, E. J. Louis, S. C. Gau, and A. G. MacDiarmid, Phys. Rev. Lett. 39, 1098 (1977).

[13] S. R. Forrest, Nature (London) 428, 911 (2004).

[14] S. Sanvito and A. R. Rocha, J. Comput. Theor. Nanosci. 3, 624 (2006).

[15] S. Pramanik, C.-G. Stefanita, S. Patibandla, S. Bandyopadhyay, K. Garre, N. Harth, and M. Cahay, Nat. Nanotechnol. 2, 216 (2007).

[16] K. Tsukagoshi, B. W. Alphenaar, and H. Ago, Nature (London) 401, 572 (1999).

[17] G. Szulczewski, S. Sanvito, and J. M. D. Coey, Nat. Mater. 8, 693 (2009).

[18] B. Dieny, V. Speriosu, B. Gurney, S. Parkin, D. Wilhoit, K. Roche, S. Metin, D. Peterson, and S. Nadimi, J. Magn. Magn. Mater. 93, 101 (1991).

[19] I. Žutić, J. Fabian, and S. Das Sarma, Rev. Mod. Phys. 76, 323 (2004).

[20] V. I. Krinichnyi, Synth. Met. 108, 173 (2000).

[21] S. Bandyopadhyay, Phys. Rev. B 81, 153202 (2010).

[22] A. J. Drew et al., Nat. Mater. 8, 109 (2009).
[23] F. J. Wang, C. G. Yang, Z. V. Vardeny, and X. G. Li, Phys. Rev. B 75, 245324 (2007).

[24] V. Dediu, L. E. Hueso, I. Bergenti, A. Riminucci, F. Borgatti, P. Graziosi, C. Newby, F. Casoli, M. P. De Jong, C. Taliani, and Y. Zhan, Phys. Rev. B 78, 115203 (2008).

[25] C. Cohen-Tannoudji, B. Diu, and F. Laloè, Quantum Mechanics. 2, Textbook physics (John Wiley and Sons, New York 1977).

[26] A. Troisi, J. Chem. Phys. 134, 034702 (2011).

[27] C. Motta and S. Sanvito, J. Chem. Theor. Comp. 10, 4624 (2014).

[28] G. H. Wannier, Phys. Rev. 52, 191 (1937).

[29] G. H. Wannier, Rev. Mod. Phys. 34, 645 (1962).

[30] N. Marzari and D. Vanderbilt, Phys. Rev. B 56, 12847 (1997).

[31] N. Marzari, A. A. Mostofi, J. R. Yates, I. Souza, and D. Vanderbilt, Rev. Mod. Phys. 84, 1419 (2012).

[32] J. M. Soler, E. Artacho, J. D. Gale, A. García, J. Junquera, P. Ordejón, and D. Sánchez-Portal, J. Phys. Condens. Matter 14, 2745 (2002).

[33] A. A. Mostofi, J. R. Yates, Y.-S. Lee, I. Souza, D. Vanderbilt, and N. Marzari, Comput. Phys. Commun. 178, 685 (2008).

[34] Z. Ning and H. Tian, Chem. Commun. 37, 5483 (2009).

[35] L. Fernández-Seivane, M. A. Oliveira, S. Sanvito, and J. Ferrer, J. Phys. Condens. Matter 18, 7999 (2006).

[36] The correctness of the elements $U_{p m}^{s}(\mathbf{k})$ and $e^{i \mathbf{k} \cdot \mathbf{R}}$ is easily verified by ensuring that the following relation is satisfied:

$$
\begin{aligned}
\left\langle w_{m \mathbf{R}_{1}} \mid w_{n \mathbf{R}_{2}}\right\rangle & =\frac{1}{N} \sum_{p} \int_{\mathrm{FBZ}} d \mathbf{k}\left\langle w_{m \mathbf{R}_{1}} \mid \psi_{p \mathbf{k}}\right\rangle\left\langle\psi_{p \mathbf{k}} \mid w_{n \mathbf{R}_{2}}\right\rangle \\
& =\frac{1}{N} \sum_{p} \int_{\mathrm{FBZ}} d \mathbf{k} U_{p m}^{*}(\mathbf{k}) U_{p n}(\mathbf{k}) e^{i \mathbf{k} \cdot\left(\mathbf{R}_{1}-\mathbf{R}_{2}\right)} \\
& =\delta_{m, n} \delta_{\mathbf{R}_{1}, \mathbf{R}_{2}} .
\end{aligned}
$$

[37] The real spherical harmonics are constructed from the complex ones $|l, m\rangle$ as $|l, M\rangle=\frac{1}{\sqrt{2}}\left[|l, m\rangle+(-1)^{m}|l,-m\rangle\right]$ and $|l,-M\rangle=\frac{1}{i \sqrt{2}}\left[|l, m\rangle-(-1)^{m}|l,-m\rangle\right]$. For $M=0$ the real and complex spherical harmonics coincide.

[38] S. Romanov, J. Phys. Condens. Matter 5, 1081 (1993). 
[39] E. Moulin, F. Niess, M. Maaloum, E. Buhler, I. Nyrkova, and N. Giuseppone, Angew. Chem. Int. Ed. 49, 6974 (2010).

[40] V. Faramarzi, F. Niess, E. Moulin, M. Maaloum, J.-F. Dayen, J.-B. Beaufrand, S. Zanettini, B. Doudin, and N. Giuseppone, Nat. Chem. 4, 485 (2012).
[41] A. Akande, S. Bhattacharya, T. Cathcart, and S. Sanvito, J. Chem. Phys. 140, 074301 (2014).

[42] S. Bhattacharya, A. Akande, and S. Sanvito, Chem. Commun. 50, 6626 (2014). 